\title{
Photobiomodulation therapy as a high potential treatment modality for COVID-19
}

\author{
Sepideh Soheilifar ${ }^{1}$ (D) $\cdot$ Homa Fathi $^{2}$ (D) Navid Naghdi ${ }^{3}$ (D)
}

Received: 5 August 2020 / Accepted: 17 November 2020 / Published online: 25 November 2020

(C) Springer-Verlag London Ltd., part of Springer Nature 2020

\begin{abstract}
COVID-19 is now a worldwide concern, causing an unprecedented pandemic. The infected cases show different symptoms based on the severity of the disease. In asymptomatic and non-severe symptomatic cases, the host immune system can successfully eliminate the virus and its effects. In severe cases, however, immune system impairment causes cytokine release syndrome which eventually leads to acute respiratory distress syndrome (ARDS). In recent years, photobiomodulation (PBM) has shown promising results in reducing acute pulmonary inflammation. Considering the high potential impact of PBM on immune responses, we hypothesized that using PBM could be an effective treatment modality for ARDS management in COVID-19 patients.
\end{abstract}

Keywords Coronavirus · Low-level light therapy $\cdot$ Respiratory distress syndrome, adult $\cdot$ Inflammation $\cdot$ COVID-19 . Photobiomodulation

\section{Background}

In the final days of 2019, China reported the emergence of an unknown pathogen causing pneumonia-like symptoms in the infected cases in Wuhan, Hubei. On January 7, 2020, Chinese Center for Disease Control and Prevention detected the origin as a novel virus from the Coronaviridae family. World Health Organization (WHO) soon confirmed that human to human transmission of the virus has led to a worldwide "pandemic" [1]. The virus was named severe acute respiratory syndrome coronavirus 2 (SARS-CoV-2) causing Coronavirus disease 2019 (COVID-19).

Coronaviridae is a large family of enveloped, positivesense, single-stranded RNA virus [2]. Based on the genome structure and phylogenetic relationships, this family is further categorized into four groups; Alphacoronavirus, Betacoronavirus, Gammacoronavirus, and Deltacoronavirus. Alpha and Betacoronaviruses are specific to mammals and

Navid Naghdi

3n.navid.naghdi@gmail.com

1 Orthodontics Department, Dental Implant Research Center, Dental faculty, Hamadan University of Medical Sciences, Hamadan, Iran

2 Dentistry Faculty, McGill University, Montreal, Quebec, Canada

3 Oral and Maxillofacial Surgery Department, Dental Implant Research Center, Dental Faculty, Hamadan University of Medical Sciences, Shahid Fahmideh street, Hamadan 654178-38741, Iran cause respiratory diseases in humans, namely Severe Acute Respiratory Syndrome coronavirus (SARS-CoV) and the Middle East Respiratory Syndrome coronavirus (MERS$\mathrm{CoV}$ ). Delta and Gammacoronaviruses cause infection in both mammals and birds [3]. Genetic analysis revealed that SARSCoV-2 is most probably in the Betacoronavirus category [4].

The infected cases show different symptoms based on the severity of the disease. In asymptomatic and non-severe symptomatic cases, the host immune system can successfully eliminate the virus and its effects. In severe cases, however, immune system impairment causes cytokine release syndrome which eventually leads to acute respiratory distress syndrome (ARDS) [5].

One of the treatment strategies is to eliminate inflammatory response in the host. Although some medications such as immunosuppressants have this effect, they cause delayed body response to virus elimination [6]. To address this issue, alternative treatment modalities for inflammation elimination are urgently needed. One such potential treatment is photobiomodulation (PBM), also known as low-level laser therapy (LLLT). PBM is an alternative modality for local management of increased inflammation, which has been used from 50 years ago [7]. It is defined as a low power laser or lightemitting diode (LED) in the range of $1-500 \mathrm{~mW}$ utilized to promote tissue regeneration and decrease inflammation and pain. A narrow spectral width light in red or near infra-red range $(600-1000 \mathrm{~nm})$ with $1-5000 \mathrm{~mW} / \mathrm{cm}^{2}$ power density is used in PBM [8]. PBM can change cellular and molecular metabolism, 
signaling, inflammation, and chemical messenger release. It has shown promising results in reducing acute pulmonary inflammation, as they have a high potential for the local balance of immune responses [9]. Therefore, the objective of this paper is to hypothesize that using PBM could be an effective treatment modality for ARDS management in COVID-19 patients.

\section{COVID-19}

COVID-19 is a new form of coronavirus with phylogenetic similarities to SARS-CoV [10]. COVID-19 infection occurs in three forms:

1. Asymptomatic incubation form (the virus detection is probable)

2. Non-severe symptomatic form (the virus detection is definite)

3. Severe symptomatic form with high viral contamination (the virus detection is definite)

The immune response against COVID-19 occurs in two clinical phases:

1- Specific adaptive immune responses: These responses occur as a result of B/T cells stimulation and immunoglobulin secretion. (IgM and IgG) [11]. They are aimed at virus elimination and disease de-escalation and occur in the first and second forms of the disease. The patient's overall wellbeing and genetic background affects the specific adaptive immune responses. For instance, efficient immune system could constrict virus propagation and tissue destruction. In addition, it seems that patients with specific Human Leukocyte Antigen haplotypes are more susceptible to this viral infection. In this phase, treatment strategies mainly include the improvement of the immune system.

2- Severe inflammatory responses: These severe responses occur as a result of uncontrolled amplified immune response leading to sudden and severe release of cytokines which is called cytokine storm. They happen in the lungs and are the leading causes of mortality in the third form of the disease. Some patients may suffer from acute respiratory distress syndrome (ARDS) at this phase, which can cause pulmonary edema, lung failure, and dysfunction of the liver, heart, and kidney. Increased serum levels of IL$1 \beta$ (interleukin-1 beta), IL-2, IL-7, IL-8, IL-9, IL-10, IL17, G-CSF (granulocyte colony-stimulating factor), GMCSF (granulocyte-macrophage colony-stimulating factor), IFN $\gamma$ (immune interferon gamma), TNF $\alpha$ (tumor necrosis factor alpha), IP10 (induced protein 10), MCP1 (monocyte chemoattractant protein 1), MIP1A (macrophage inflammatory protein 1A), and MIP1B may also be observed [12]. Therefore, treatment modalities mainly focus on immunosuppressive strategies and symptom management at this phase [5].

\section{Treatment protocol}

Treatment protocols can be generally categorized into four groups:

1. Preventing virus entrance or fusion to the host cell

2. Preventing replication of the virus's RNA in the host cell

3. Boosting the host immune system

4. Suppression of the severe inflammatory reaction in the lungs

Li et al. recommended that the treatment focus should be on the first and third protocols in the initial phase of clinical response. In the severe phase of the immune response, however, suppression of pulmonary inflammation and preventing ARDS are prioritized [13].

The mortality rate of COVID-19 ARDS is high [14]. Immunosuppressive drugs such as corticosteroids may be of choice for inhibiting severe immune response in ARDS. However, they may delay the body response to virus elimination [6]. Besides, a systematic review and meta-analysis showed that prescription of corticosteroids in influenza pneumonia patients leads to increased mortality rate, secondary infection rate, and longer hospitalization period [15].

\section{Anti-inflammatory effects of PBM}

One way to suppress the severe inflammatory reaction in the lung (the fourth treatment protocol) is PBM. Antiinflammatory and regenerative effects of PBM have been observed in the treatment of allergic lung inflammation, vocal fold injuries, periodontitis, and oral lesions [16-19]. In comparison with medications, such as immunosuppressive drugs including corticosteroids PBM has mostly local effects, and its systemic effects are limited. In the following section, we will discuss how utilizing PBM could potentially be a treatment modality for COVID-19 patients.

\section{Effect of PBM on lung inflammation}

Several studies have reported the positive effects of PBM on lung inflammatory diseases. de Lima et al. assessed the effect of PBM on ARDS in mice. They observed that TNF $\alpha$ has a tremendous effect on neutrophil sequestration and migration, which plays a key role in ARDS pathogenesis. PBM successfully decreased the neutrophilic influx and TNF $\alpha$ levels in bronchoalveolar lavage fluid (BALF) and increased cAMP 
and decreased TNF $\alpha$ m-RNA in alveolar macrophage. These events reduced the incidence of ARDS [9].

Oliveira et al. evaluated the effect of PBM on ARDS in mice models. Based on the findings, the severity of the disease is positively correlated to activation and apoptosis rate of neutrophils. PBM significantly reduced the amount of neutrophilic migration to the lung tissue and eventually decreased the severity of the disease [20].

Mehani compared the immunomodulatory effects of PBM and inspiratory physical therapy in chronic obstructive pulmonary disease (COPD) patients. Both methods were reported to be clinically useful; however, PBM was more effective in decreasing IL-6 level and increasing CD4+/CD8+ proportion [21].

Other experimental and clinical investigations also reported the positive effects of PBM on acute and chronic pulmonary inflammation, and acute pulmonary inflammation induced by intestinal ischemic-reperfusion [22-24]. Also, it has been observed that PBM may be useful for decreasing pulmonary fibrosis [25].

\section{PBM mechanism in acute pulmonary inflammation}

Acute pulmonary inflammation is related to the increased number of polymorphonuclear neutrophils (PMN) in interstitial space and release of some pro-inflammatory cytokines, including IL-1 $\beta$, IL-6, IL-8, TNF $\alpha$, MCP-1, and MIP-1. Around 24$48 \mathrm{~h}$ after these events take place, cytokine concentration returns to normal in bronchoalveolar lavage fluid; however, the number of PMNs, monocytes, macrophages, and lymphocytes rise, and pulmonary dysfunction occurs because of collagen deposition. Several studies reported that PBM might play a substantial role in managing all the aforementioned immune responses, including the increased number of PMNs, monocytes, and macrophages; the release of pro-inflammatory cytokines; and collagen deposition. In addition to the inhibitory effect on inflammatory cell chemotaxis, PBM can also decrease the number of present leukocytes in inflammation sites [23].

Increased level of IL- $1 \beta$ is concomitant with poor prognosis in ARDS patients. IL- $1 \beta$ has a central role in the initiation of inflammatory processes [20]. This cytokine also increases neutrophil survival rate and makes inflammation persistent. PBM can reduce the incidence and severity of ARDS by decreasing the IL- $1 \beta$ level. IL- 6 , a pleiotropic cytokine, has a key role in ARDS pathophysiology and is related to the poor prognosis of the disease. ARDS patients show an increased level of IL- 6 in the lungs and plasma. PBM has been able to decrease IL-6 levels both in the lungs and plasma in ARDS mice $[20,23]$. IL- 8 contributes to ARDS pathophysiology, neutrophil chemotaxis, and survival in the lung. PBM can significantly decrease the level of IL-8 in the lung, attenuate ARDS, and decrease its mortality rate [20].
$\mathrm{TNF} \alpha$ is responsible for adhesion and activation of neutrophils, coagulation, and edema. It can also stimulate IL-6 release. TNF $\alpha$ level is usually high in the serum and lung of ARDS patients. PBM has been shown to be useful in decreasing TNF $\alpha$ levels at both sites [20,23]. MCP-1 has a crucial role in recruiting monocytes, and its level increases in pulmonary inflammation. Monocyte migration can be reduced following PBM due to MCP-1 decrease [23].

On the other hand, the role of IL-10 is not still clear. Some authors reported it as a predictor of poor prognosis [26], while the others know it as a regulatory cytokine that is also released during the cytokine storm. Its role is to limit the immune response to pathogens and restrict host cellular damage [27]. Several studies have stated that an imbalance between the level of TNF $\alpha$ and IL-10 increases host cellular damage and complications. PBM can increase the level of IL-10 and improve the balance of inflammatory processes $[8,24]$.

\section{Other effects of PBM}

Another theory in COVID-19 pathophysiology is the role of decreased number of CD4+ and CD8+ T cells and the lymphocytic imbalance. $\mathrm{T}$ cells play a crucial role in the immune response against the viral pathogens; $\mathrm{CD} 4+$ helper $\mathrm{T}$ cells guide $\mathrm{B}$ cells, and cytotoxic $\mathrm{T}$ cells and $\mathrm{CD} 8+$ cytotoxic $\mathrm{T}$ cells eliminate viral pathogens by releasing molecules such as perforins, granzymes, and IFN $\gamma$ [28]. PBM is shown to be capable of increasing CD4+ and CD8+ T cells and improving the balance between them [21].

In some cases of ARDS, a delayed increase of macrophages can worsen the situation. In Cury et al. study, PBM decreased this delayed inflammation phase, as well [23].

While PBM has been widely used to improve healing, potential negative outcomes have also been observed. For instance, PBM could induce fibroblastic migration, which in turn causes collagen deposition in the lung tissues; this could eventually lead to pulmonary fibrosis. However, previous studies have declined this harmful effect in humans and animals $[21,23]$. Besides, de Brito et al. have stated that PBM may even have an anti-fibrotic effect by decreasing TGF $\beta$ (transforming growth factor beta) in the fibroblast cells and lung tissue [25].

\section{Conclusion}

Considering the pathophysiology of COVID-19 and potential positive effects of PBM in balancing the function of the immune system, this treatment modality could be effective in severe COVID-19 cases with ARDS. COVID-19 mortality is mainly because of cytokine storm in severe cases. PBM has the potential to decrease the level of pro-inflammatory cytokines such as 
IL-1 $\beta$, IL-6, IL-8, TNF $\alpha$, and MCP-1 and improve the balance of IL-10. These effects can balance immune response and decrease the impact of cytokine storm. PBM is mainly local and has very limited adverse side effects [7]. Unlike corticosteroids, it does not cause delayed body response to virus elimination, secondary infection, or longer hospitalization period.

This hypothesis is mainly based on theoretical data. The authors suggest that researchers should assess the potentials of this treatment method as it might save the lives of severely affected patients.

Acknowledgments The authors would like to extend their gratitude towards Dr. Martin O. Martel, Assistant Professor of faculties of dentistry and medicine in McGill University, for giving valuable advice and insightful suggestions.

Authors' contributions Sepideh Soheilifar: Conceptualization, writingoriginal draft.

Homa Fathi: Writing — review and editing.

Navid Naghdi: Conceptualization, writing — original draft.

\section{Compliance with ethical standards}

Conflict of interest The authors declare that they have no conflict of interest.

\section{References}

1. WHO (2020) No Title. In: Coronavirus Dis. 2019 Situat. Rep. - 51. https://www.who.int/docs/default-source/coronaviruse/situationreports/20200311-sitrep-51-covid-19.pdf?sfvrsn=1ba62e57_10. Accessed 11 Mar 2020

2. Phan MVT, Ngo Tri T, Hong Anh P, et al (2018) Identification and characterization of Coronaviridae genomes from Vietnamese bats and rats based on conserved protein domains. Virus Evol 4:vey035

3. Kumar S, Maurya VK, Prasad AK et al (2020) Structural, glycosylation and antigenic variation between 2019 novel coronavirus (2019$\mathrm{nCoV}$ ) and SARS coronavirus (SARS-CoV). VirusDisease 1-9

4. Ortiz-Prado E, Simbaña-Rivera K, Gómez-Barreno L et al (2020) Clinical, molecular, and epidemiological characterization of the SARS-CoV-2 virus and the Coronavirus Disease 2019 (COVID19), a comprehensive literature review. Diagn Microbiol Infect Dis 98:115094. https://doi.org/10.1016/j.diagmicrobio.2020.115094

5. Shi Y, Wang Y, Shao C et al (2020) COVID-19 infection: the perspectives on immune responses

6. Favalli EG, Ingegnoli F, De Lucia O et al (2020) COVID-19 infection and rheumatoid arthritis: faraway, so close! Autoimmun Rev 102523

7. Mussttaf RA, Jenkins DFL, Jha AN (2019) Assessing the impact of low level laser therapy (LLLT) on biological systems: a review. Int J Radiat Biol 95:120-143

8. Carvalho JL, Britto A, de Oliveira APL et al (2017) Beneficial effect of low-level laser therapy in acute lung injury after iI/R is dependent on the secretion of IL-10 and independent of the TLR/ MyD88 signaling. Lasers Med Sci 32:305-315

9. de Lima FM, Moreira LM, Villaverde AB et al (2011) Low-level laser therapy (LLLT) acts as cAMP-elevating agent in acute respiratory distress syndrome. Lasers Med Sci 26:389-400

10. Guan W, Ni Z, Hu Y et al (2020) Clinical characteristics of coronavirus disease 2019 in China. N Engl J Med
11. Li X, Geng M, Peng Y et al (2020) Molecular immune pathogenesis and diagnosis of COVID-19. J Pharm Anal

12. Wu D, Yang XO (2020) TH17 responses in cytokine storm of COVID-19: an emerging target of JAK2 inhibitor fedratinib. J Microbiol Immunol Infect

13. Li H, Zhou Y, Zhang M et al (2020) Updated approaches against SARS-CoV-2. Antimicrob Agents Chemother

14. Yang $\mathrm{X}, \mathrm{Yu} \mathrm{Y,Xu} \mathrm{J}$ et al (2020) Clinical course and outcomes of critically ill patients with SARS-CoV-2 pneumonia in Wuhan, China: a single-centered, retrospective, observational study. Lancet Respir Med

15. Ni Y-N, Chen G, Sun J et al (2019) The effect of corticosteroids on mortality of patients with influenza pneumonia: a systematic review and meta-analysis. Crit Care 23:99

16. Lavaee F, Shadmanpour M (2019) Comparison of the effect of photodynamic therapy and topical corticosteroid on oral lichen planus lesions. Oral Dis

17. Costa Carvalho JL, De Brito AA, De Oliveira APL et al (2016) The chemokines secretion and the oxidative stress are targets of lowlevel laser therapy in allergic lung inflammation. J Biophotonics 9: 1208-1221

18. Gandhi KK, Pavaskar R, Cappetta EG, Drew HJ (2019) Effectiveness of adjunctive use of low-level laser therapy and photodynamic therapy after scaling and root planing in patients with chronic periodontitis. Int J Periodontics Restor Dent 39:837-843

19. Lou Z, Zhang C, Gong T et al (2019) Wound-healing effects of 635-nm low-level laser therapy on primary human vocal fold epithelial cells: an in vitro study. Lasers Med Sci 34:547-554

20. Oliveira MC Jr, Greiffo FR, Rigonato-Oliveira NC et al (2014) Low level laser therapy reduces acute lung inflammation in a model of pulmonary and extrapulmonary LPS-induced ARDS. J Photochem Photobiol B Biol 134:57-63

21. Mehani SHM (2017) Immunomodulatory effects of two different physical therapy modalities in patients with chronic obstructive pulmonary disease. J Phys Ther Sci 29:1527-1533

22. da Cunha Moraes G, Vitoretti LB, de Brito AA, et al (2018) Lowlevel laser therapy reduces lung inflammation in an experimental model of chronic obstructive pulmonary disease involving P2X7 receptor. Oxid Med Cell Longev 2018

23. Cury V, de Lima TM, Prado CM et al (2016) Low level laser therapy reduces acute lung inflammation without impairing lung function. J Biophotonics 9:1199-1207

24. de Lima FM, Vitoretti L, Coelho F et al (2013) Suppressive effect of low-level laser therapy on tracheal hyperresponsiveness and lung inflammation in rat subjected to intestinal ischemia and reperfusion. Lasers Med Sci 28:551-564

25. de Brito AA, da Silveira EC, Rigonato-Oliveira NC et al (2020) Low-level laser therapy attenuates lung inflammation and airway remodeling in a murine model of idiopathic pulmonary fibrosis: relevance to cytokines secretion from lung structural cells. J Photochem Photobiol B Biol 203:111731

26. Han H, Ma Q, Li C et al (2020) Profiling serum cytokines in COVID-19 patients reveals IL-6 and IL-10 are disease severity predictors. Emerg Microbes Infect 9:1123-1130. https://doi.org/ $10.1080 / 22221751.2020 .1770129$

27. Iyer SS, Cheng G (2012) Role of interleukin 10 transcriptional regulation in inflammation and autoimmune disease. Crit Rev Immunol 32

28. Diao B, Wang C, Tan Y et al (2020) Reduction and functional exhaustion of T cells in patients with coronavirus disease 2019 (COVID-19). Medrxiv

Publisher's note Springer Nature remains neutral with regard to jurisdictional claims in published maps and institutional affiliations. 